\title{
Maternal killer-cell immunoglobulin-like receptors and paternal human leukocyte antigen ligands in recurrent pregnancy loss cases in Turkey
}

\author{
Mehmet Onur Elbaşı ${ }^{1, *}$, Aysın Tulunay ${ }^{2}$, Hale Karagözoğlu ${ }^{3}$, Semra Kahraman ${ }^{4}$, Emel Ekşioğlu-Demiralp ${ }^{1, *}$ \\ ${ }^{1}$ Tissue Typing and Immunology Laboratory, Istanbul Memorial Hospital, Istanbul; ${ }^{2}$ Department of Immunology, Marmara University, School of \\ Medicine, Istanbul; ${ }^{3}$ Karagözoğlu Clinic for Obstetrics and Gynecology and IVF, Istanbul; ${ }^{4}$ In Vitro Fertilization Unit, Istanbul Memorial Hospital, Istanbul, \\ Turkey
}

Objective: The survival of a semi-allogeneic fetus depends on several immunological mechanisms, and it has been suggested that recurrent pregnancy loss (RPL) could develop as a result of one or more immunological abnormalities.

Methods: Compatibility between partners for human leukocyte antigen (HLA) genotypes and the relationships between maternal killer-cell immunoglobulin-like receptor (KIR) and paternal HLA-Bw4/Bw6 and HLA-C1/C2 supra-groups were investigated in 25 couples with RPL in comparison to healthy couples with children. HLA and KIR genotyping was performed using polymerase chain reaction with sequence-specific primers and/or sequence-specific oligonucleotides.

Results: HLA class I incompatibility between partners, especially in HLA-B alleles, was more common in the RPL group ( $p=0.01)$. HLA-C2 homozygosity was more frequent in the male partners of RPL couples than in other groups $(p=0.03)$. The KIR2DL5 gene frequency was significantly higher in both the female and male partners of RPL couples, whereas the KIR2DS3 gene frequency in male partners of RPL couples was significantly reduced $(p=0.03)$. The presence of KIR2DL3 in women with RPL was correlated with the presence of HLA-C2 alleles in their spouses $(p=0.03)$.

Conclusion: Our data from a Turkish population suggest that male HLA-C2 homozygosity may play an important role in RPL. Additionally, an incidental match between male HLA-C2 and female HLA-C1 ligand KIR receptors might perturb the balance between activatory and inhibitory KIR-ligand interactions during pregnancy in couples affected by RPL. The roles of orphan KIR2DL5 and orphan KIR2DS3 in RPL remain obscure.

Keywords: HLA C2; Human leukocyte antigen; Killer-cell immunoglobulin-like receptor genotyping; KIR2DL5; KIR2DS3; Recurrent pregnancy loss

\section{Introduction}

Spontaneous abortion is defined as occurring when a pregnancy

Received: August 1, 2019· Revised: October 28, 2019. Accepted: January 2, 2020 Corresponding author: Emel Ekşioğlu-Demiralp

Tissue Typing and Immunology Laboratory, Istanbul Memorial Hospital, Piyalepaşa Blv, Memorial Hospital C Blok Kat 11, Okmeydanı Şişli 34385, Istanbul, Turkey Tel:+90-2123146666 Fax:+90-2123146625 E-mail:emel.demiralp@memorial.com.tr

*These authors contributed equally to this work.

**This project was partly supported by the Marmara University Scientific Research Projects Commission (No. SAG-C-YLP-060308-0035).

This is an Open Access article distributed under the terms of the Creative Commons Attribution Non-Commercial License (http://creativecommons.org/licenses/by-nc/4.0/) which permits unrestricted non-commercial use, distribution, and reproduction in any medium, provided the original work is properly cited. ends before the 24th week of gestation without any mechanical or pharmacological interference. Recurrent pregnancy loss (RPL) refers to two or more consecutive miscarriages clinically recognized before 24 weeks after the last date of menstruation [1]. Research has shown that miscarriages occur in roughly $15 \%$ of clinically identified pregnancies [2]. Miscarriages that recur with no apparent cause are encountered in $1 \%$ of the population [3]. Even after a complete evaluation, a likely cause cannot be determined in almost half of the cases [4]. The causes of RPL can be genetic, endocrinological, anatomical, immunological, and microbiological, with considerable variation among individuals [5].

During pregnancy, it is vital for the fetus that both the mother and the fetus have a properly functioning immune system. Because of 
paternal genes, embryonic tissues and the tissues surrounding the embryo can be recognized as an allograft by the mother. Some researchers have proposed that human leukocyte antigen (HLA) matching between partners can cause disruption of immune recognition by trophoblasts, resulting in miscarriages. However, it has not been proven that HLA matching causes miscarriages. On the contrary, in pregnancies that occurred through oocyte donation, it has been shown that more HLA matches between the mother and fetus were associated with a healthier progression of the pregnancy $[6,7]$. The fact that syncytiotrophoblast cells of the chorionic villi do not express HLA antigens is an important factor in preventing the rejection of semi-allogeneic antigens [8]. On the surface of extravillous cytotrophoblast cells, the classical major histocompatibility complex (MHC) class I product (HLA-C) is found, as well as the nonclassical MHC class I antigens HLA-E and HLA-G [9-11]. MHC genes contribute to recurrent miscarriages, as has been reported by various groups in several studies [12-14]. Even though they are not found in extravillous cytotrophoblast cells, it has been proposed that HLA-B and HLADQB1 alleles might be related to $\operatorname{RPL}[15,16]$.

Although the thymus undergoes dramatic changes during pregnancy; it is known that the numbers of maternal $\mathrm{T}$ cells, B cell, natural killer (NK) cells, and their subgroups in peripheral blood do not change. However, T cell function diminishes $[17,18]$. In comparison to peripheral blood, the distribution of lymphocytes is different in the uterus. NK cells account for $70 \%$ of uterine lymphocytes [11]. NK cells recognize cells with no $\mathrm{MHC}$ on their surface and then eliminate them through their cytotoxic activity $[19,20]$. Killer-cell immunoglobulin-like receptors (KIRs) exert inhibitory and activating effects on NK cell cytotoxicity [21]. HLA-C molecules, which are KIR ligands, are separated into two groups (C1 and C2) based whether lysine or asparagine is present at the 80th position on the $\mathrm{a} 1$ helix of the heavy chain [22,23]. The known ligands of HLA-C1 are KIR2DL2, KIR2DL3, and KIR2DS2, and the known ligands of HLA-C2 are KIR2DL1 and KIR2DS1 [23-25]. Some KIR ligands, such as KIR2DS3 and KIR2DL5, have not been completely described and are defined as orphan genes $[26,27]$.

Similar to HLA-C1/C2, HLA-B molecules are subdivided into two supra-groups (Bw4 and Bw6) based on the existence of two different epitopes in the 77-83 position on the a1 helix of the heavy chain [28]. Even though KIRs mainly interact with HLA-C molecules, interactions among HLA-Bw4/Bw6 and KIR molecules have been emphasized in various studies. Some KIRs, such as KIR3DL1, can interact with some HLA-B groups [29]. KIR3DL1 engages in complex interactions, especially with the HLA-Bw4 supra-group [30,31]. Because of the variability of KIR3DL1 allotypes and Bw4 epitope specificity, KIR3DL1-positive NK cells might not recognize all Bw4+ molecules. This diversity and specificity may play an effective role in promoting graft acceptance [31]. Indeed, the impact of KIR3DL1/3DS1 alleles and HLA-Bw4 interactions on allograft survival has been reported [29]. Although the functions of activatory and inhibitory KIR molecules have been identified, more data is needed to interpret the clinical implications of maternal KIR and fetal HLA interactions during pregnancy [32].

In light of previous data, we examined KIR and HLA class I and II groups in Turkish couples experiencing RPL to understand whether matching status between couples plays a role in RPL and/or whether the relationship between partners' KIR genes and HLA-C1/C2 and HLA-Bw4/6 status differs between couples experiencing RPL and healthy couples.

\section{Methods}

\section{Patients}

Patients for this study were chosen among those who presented to the Memorial Hospital In Vitro Fertilization Unit suffering from spontaneous abortion. Couples with at least 3 and at most 6 miscarriages, and with no successful pregnancies, participated in this study. The other inclusion criteria for participation in the study were negative cervical mucus culture results (Chlamydia/Ureaplasma); normal karyotype analysis results; no hormonal anomalies such as hyperprolactinemia or hyperandrogenemia; no history of diagnosed endocrine diseases such as diabetes, hyperthyroidism, or hypothyroidism; negative antinuclear antibody and anticardiolipin antibody results, and normal semen analysis results. In total, 25 couples who satisfied the above criteria, whose recurrent miscarriages could not be explained by any of the above possibilities, and who were diagnosed with RPL were included. The control group comprised 24 healthy couples with children for HLA typing and 39 healthy couples for KIR genotyping who had not experienced miscarriages. Additionally, our HLA series from healthy individuals who had applied to be transplant donors was used for the HLA comparisons (unpublished data, $\mathrm{n}=1,528$ ). The study was approved by Ethics Committee of Marmara University, Faculty of Medicine (No. B.30.2MAR.0.01.02/AEK/ MARYÇ-2007-0078). Informed consent was obtained from the participants before the study.

\section{DNA isolation}

Genomic DNA was isolated from $5 \mathrm{~mL}$ of venous blood with EDTA (ethylenediaminetetraacetic acid) using the silica bead-based isolation method modified by Eksioglu Demiralp et al. [33]. The purity of isolated DNA samples was measured using spectrophotometry (EPOCH; BioTek, Winooski, VN, USA). 


\section{HLA typing}

Individual HLA-A, -B, -DR, and -DQ tissue typing was performed by low-resolution polymerase chain reaction with sequence-specific primers (PCR-SSP) or sequence-specific oligonucleotides (PCR-SSO) using the Luminex platform (OneLambda Inc., West Hills, CA, USA). High-resolution bead-based multiplex PCR-SSO (OneLambda Inc.) was used for HLA-C typing. The Bw4 and Bw6 supra-group analyses were conducted based on the common antigen list found on the allelefrequencies.net website [34]. The analysis of the HLA-C1 and HLAC2 groups with their KIR counterparts was conducted based on a previously published list [22]. The results, except for the evaluation templates, were assessed using the dedicated HLA Fusion software (OneLambda Inc.).

\section{KIR allele typing}

To determine individual-level KIR allele polymorphisms, a PCR-SSO bead-based multiplex kit was used (OneLambda Inc.). The KIR genotypes examined were 2DL1, 2DL2, 2DL3, 2DL5, 2DS1, 2DS2, 2DS3, 2DS4, 2DS5, 3DL1, and 3DS2. They were evaluated using the HLA Fusion software. The KIR gene frequencies were calculated by directly counting positive and negative loci, and KIR gene haplotypes were determined. Our KIR genotype data from healthy controls are available in the allele frequencies.net website with the name "Turkey KIR pop 3" [35].

\section{Statistics}

Gene allele frequencies were calculated by standard counting, using Microsoft Excel with the GenAlEX extension [36]. The statistical tests used were the Fisher exact chi-square test and/or the Pearson rank correlation using SPSS 14.0 (SPSS Inc., Chicago, IL, USA) or GraphPad
Prism (GraphPad Software, San Diego, CA, USA) as appropriate, and $p<0.05$ were considered to indicate statistical significance.

\section{Results}

The HLA-A, $-B,-C,-D R$, and -DQ allele frequencies in the male and female partners of couples experiencing RPL ( $n=25$ each) were compared to our data from the general Turkish population (unpublished data, $n=1,528$ ), and the HLA allele frequencies of the RPL couples did not differ significantly from those of the general population.

\section{HLA class I and class II compatibility between partners 1) Mismatching of $\mathrm{HLA}-\mathrm{A} / \mathrm{B} / \mathrm{C} / \mathrm{DR} / \mathrm{DQ}$ was more likely in $\mathrm{RPL}$ couples}

HLA matching status was compared between couples diagnosed with RPL and healthy couples. Allele compatibility frequencies and the percentages of $10 \mathrm{HLA}$ alleles (HLA-A, -B, -C, -DR, -DQ) were analyzed between the couples. According to the compatibility of both class I and II HLA alleles, the RPL and control couples were dichotomized into those compatible for 0-4 alleles and those compatible for 5-10 alleles. In RPL couples, a match rate of 0-4 alleles was significantly more common for the HLA class I and II alleles ( $96 \%$ and $68 \%$, respectively) than a match rate of $5-10$ (4\% and $32 \%$, respectively; $p=0.02$ ). Therefore, RPL couples were found to be show a predominant pattern of incompatibility (Figure 1A). The incompatibility of class I and II alleles was separately evaluated, and it was found that class I alleles were the primary source of incompatibility. Couples were categorized according to their matching status of class I alleles by grouping them as those with 0-1 match and those with 2-6 matches. Slightly more than half $(51.7 \%)$ of the healthy couples had
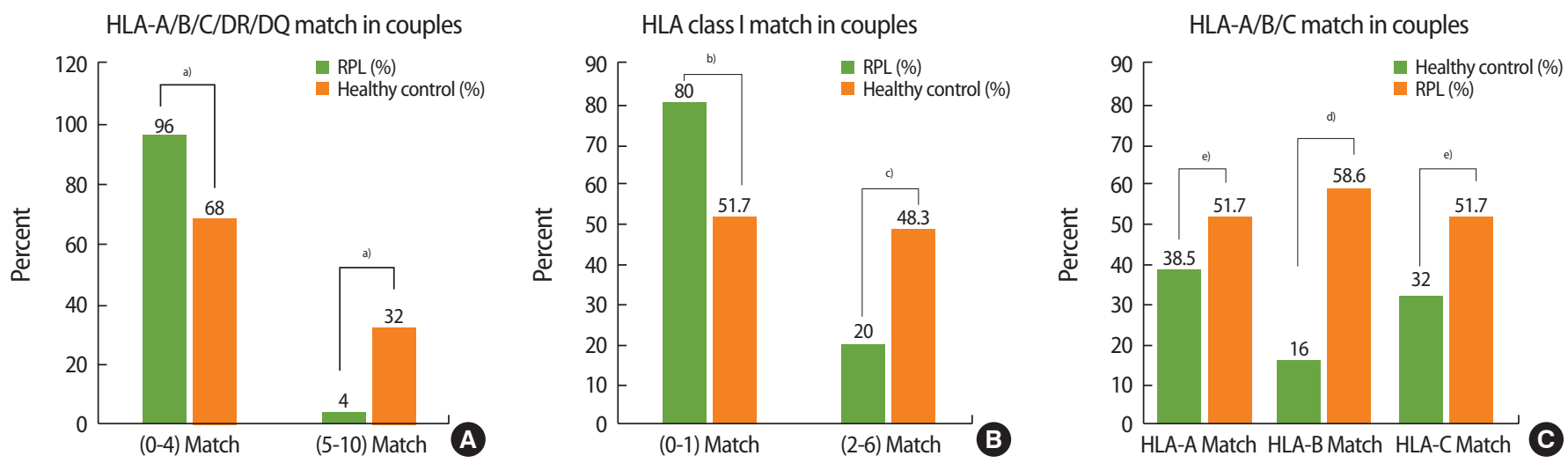

Figure 1. (A) Human leukocyte antigen (HLA)-A/B/C/DR/DQ compatibility between couples with recurrent pregnancy loss (RPL) and healthy controls. Incompatibility was more prominent in RPL couples than in couples with children. Intergroup differences were observed for both the presence of $0-4$ and $5-10$ allele matches. ${ }^{\text {a) }} p=0.02$. (B) Compatibility distributions in class I alleles in healthy and RPL couples. Incompatibility in class I alleles was more predominant in RPL couples. ${ }^{\mathrm{b})} p=003 ;{ }^{\mathrm{c})} p=0.04$. (C) Compatibility for each HLA-A/B/C allele in healthy and RPL couples. Incompatibility in the class I alleles in RPL couples was mainly due to incompatibility in HLA-B alleles. ${ }^{\mathrm{d}} p=0.001 ;{ }^{\mathrm{e}} \mathrm{NS}, p>0.05$. 
0-1 match for HLA class I alleles, while this was the case for $80 \%$ of the couples with RPL $(p=0.03)$. In contrast, $48.3 \%$ of the healthy couples had 2-6 matching HLA class I alleles, whereas this was only the case for $20 \%$ of the couples with RPL $(p=0.04)$. Similar to the overall results, the incompatibility in HLA class I alleles was more noticeable in RPL couples (Figure 1B). No significant difference was found in the comparisons of class II alleles. Considering their role in the continuation of pregnancy, HLA class I alleles were separately re-analyzed provided there was at least 1 match (Figure 1C). HLA-B allele compatibility between partners was $58.6 \%$ in the healthy couples and $16 \%$ in the RPL couples $(p=0.001)$. Therefore, the class I allele mismatches in the RPL couples was mainly due to HLA-B incompatibility (Figure 1C).

\section{2) Bw4/Bw6 frequencies in male partners}

Based on the findings on HLA-B incompatibility, the distribution frequencies of the HLA-Bw4 and HLA-Bw6 supra-groups were evaluated in the male partners of both groups, since these are KIR ligands. It was found that the distribution of the Bw4 and Bw6 antigens was $60 \%$ and $40 \%$ respectively, in the male partners of RPL couples, while it was $66 \%$ and $34 \%$, respectively, in the male partners of healthy couples. The corresponding distribution in the general Turkish male population was $48.9 \%$ and $51.1 \%$, respectively. No significant difference was found between the groups.

\section{3) Bw4 and Bw6 matching in couples}

Because the HLA-B mismatch between spouses was significant, ho-

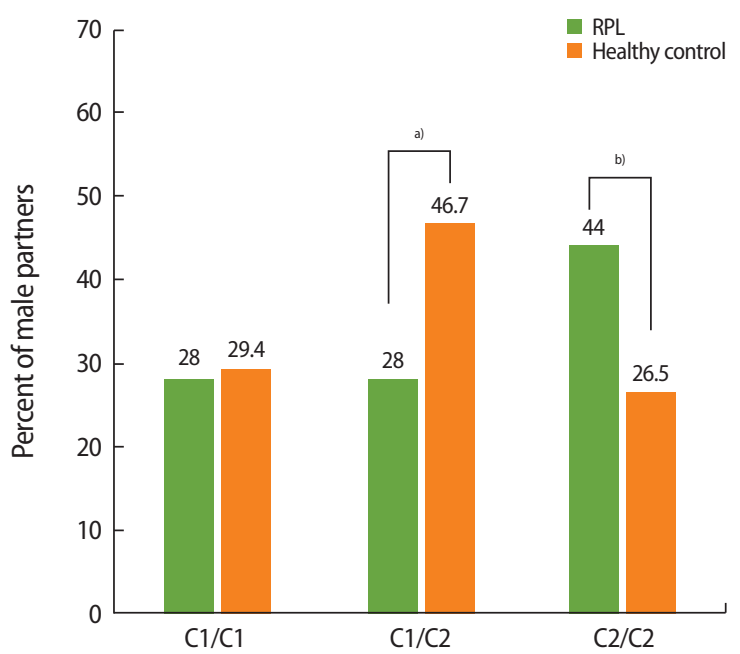

Figure 2. Human leukocyte antigen (HLA)-C1/C2 homozygosity and heterozygosity in males: when the HLA-C1/C2 groups in male partners (as killer-cell immunoglobulin-like receptor ligands) were compared between recurrent pregnancy loss (RPL) couples and healthy controls, it was found that $\mathrm{C} 2 / \mathrm{C} 2$ homozygosity was significantly

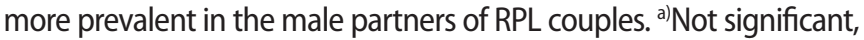
$p>0.05 ;{ }^{b)} p=0.03$. mozygosity of the Bw supra-groups in the male partners of RPL couples was evaluated in comparison with the male partners of the healthy couples and men in the general population. Although Bw6 homozygosity was slightly more common in the male partners of RPL couples, no statistically significant difference was found in either comparison. We also investigated whether the difference in HLA-B groups between the partners in RPL couples was due to incompatibility between the Bw4 and Bw6 groups. No difference was found in the distribution of the Bw4 and Bw6 groups between the male and female partners of RPL couples. Therefore, it was concluded that the differences between the Bw4 and Bw6 supra-groups, as KIR ligands, did not show statistical significance in comparisons both within the RPL couples and between the RPL and healthy couples, before KIR matching was examined.

\section{4) The frequency of male $\mathrm{C} 2$ homozygosity was higher in the RPL group}

The distribution of the HLA-C1 and HLA-C2 groups was evaluated in terms of homozygosity (C1/C1 or C2/C2) and heterozygosity (C1/ C2). The male partners of RPL couples were compared to those of healthy couples and the general Turkish population. The distribution of the $\mathrm{C} 1 / \mathrm{C} 1$ and $\mathrm{C} 1 / \mathrm{C} 2$ genotypes did not differ significantly between the male partners of RPL couples and those of healthy couples, and was found to be similar to that of the general population. Although no statistically significant difference was found in HLA-C allele compatibility within RPL couples, the frequency of C2/C2 homozygosity was significantly higher in the male partners of RPL couples (44\% in male partners of RPL couples vs. $26.5 \%$ in male partners of healthy couples; $p=0.03$ ) (Figure 2 ).

\section{KIR haplotype distributions}

The frequencies of the AA and Bx KIR haplotypes were determined in the female partners of RPL couples $(n=25)$, the male partners of RPL couples $(n=25)$, healthy women with children $(n=39)$, and members of the general Turkish population $(n=135)$. No significant differences were found in KIR haplotype frequencies between the female partners of RPL couples and either healthy controls or the general Turkish population. The AA haplotype was found in $24 \%$ of female partners of RPL couples, $33.3 \%$ of healthy women with chil-

Table 1. KIR-AA and -Bx haplotype frequencies

\begin{tabular}{lccc}
\hline Variable & $\begin{array}{c}\text { Female RPL } \\
(n=25)\end{array}$ & $\begin{array}{c}\text { Female healthy } \\
\text { control }(n=39)\end{array}$ & $\begin{array}{c}\text { Turkish population } \\
(n=135)\end{array}$ \\
\hline AA haplotype $^{\text {a) }}$ & 24 & 33.3 & 24.4 \\
Bx haplotype $^{\text {a) }}$ & 76 & 66.7 & 75.6
\end{tabular}

Values are presented as percent.

KIR, killer-cell immunoglobulin-like receptor; RPL, recurrent pregnancy loss.

${ }^{a}$ No significant difference between groups, $p>0.05$. 
Table 2. Frequencies of inhibitory and activatory KIRs in RPL and healthy control couples

\begin{tabular}{|c|c|c|c|c|c|}
\hline Variable & $\begin{array}{l}\text { Female RPL } \\
\quad(n=25)\end{array}$ & $\begin{array}{l}\text { Female healthy contro I } \\
\qquad(n=39)\end{array}$ & $\begin{array}{c}\text { Male RPL } \\
(n=25)\end{array}$ & $\begin{array}{l}\text { Male healthy control } \\
\qquad(\mathrm{n}=44)\end{array}$ & $p$-value \\
\hline \multicolumn{6}{|c|}{ Inhibitory KIR } \\
\hline 2DL2 & 52 & 41 & 56 & 43.2 & NS \\
\hline 2DL3 & 92 & 84.6 & 92 & 93.2 & NS \\
\hline $2 D L 5^{a)}$ & $70.4^{\mathrm{b}, \mathrm{c}}$ & $43.6^{\mathrm{b})}$ & $72^{\mathrm{b}, \mathrm{c}}$ & $31.8^{\mathrm{c})}$ & $0.03^{b)}, 0.001^{c}$ \\
\hline \multicolumn{6}{|c|}{ Activatory KIR } \\
\hline 2DS1 & 52 & 35.9 & 56 & 52.3 & NS \\
\hline 2DS2 & 52 & 51.3 & 56 & 52.3 & NS \\
\hline $2 D S 3^{d)}$ & 20 & $28.2^{\mathrm{b})}$ & $4^{b, c)}$ & $40.9^{c}$ & $0.02^{b}, 0.001^{c}$ \\
\hline 2DS4 & 100 & 89.7 & 100 & 90.9 & NS \\
\hline 2DS5 & 36 & 25.6 & 32 & 40.9 & NS \\
\hline 3DS1 & 40 & 25.6 & 32 & 54.5 & NS \\
\hline
\end{tabular}

Values are presented as percent.

KIR, killer-cell immunoglobulin-like receptor; RPL, recurrent pregnancy loss; NS, not significant.

${ }^{a}$ )Odds ratio for KIR2DL5: 3.07 and 5.5 for the comparison between women and men, respectively; ${ }^{\text {b)}}$ Comparison to female; ${ }^{\mathrm{c}}$ Comparison to male; ${ }^{\mathrm{d})}$ Odds ratio for KIR2DS3: 0.17 compared to female partners of RPL couples and 0.06 compared to heathy control men.

dren, and $24.4 \%$ of the general Turkish population, whereas the corresponding distribution of the Bx haplotype was $76 \%, 66.7 \%$, and $75.6 \%$, respectively $(p>0.05)$ (Table 1 ).

\section{1) Frequencies of activating and inhibitory KIR genes}

The female partners of RPL couples $(n=25)$, male partners of RPL couples $(n=25)$, healthy women $(n=39)$, and healthy men $(n=44)$ underwent KIR genotyping. The frequencies of genes responsible for expression of KIR molecules with long and short cytoplasmic tails were examined separately and compared between the groups. KIR2DL1, KIR2DL4, KIR3DL2, KIR3DL3, and the pseudo-genes (2DP1, 3DP1) found in each individual were excluded from the comparisons.

The frequency of the inhibitor KIR2DL5 - an orphan gene-was significantly higher in both the male and female partners of RPL couples than in healthy males and females ( $p=0.001$ and $p=0.03$, respectively) (Table 2). The frequency of another orphan gene, KIR2DS3, in the male partners of RPL couples was significantly lower in comparison with the male and female partners of healthy couples ( $p=0.02$ and $p=0.001$, respectively) (Table 2 ). There were no differences in the frequencies of other KIR genes between the groups. To summarize, the frequency of the KIR2DL5 gene was significantly higher in the male and female partners of RPL couples than in the normal population, and the KIR2DS3 gene frequency was significantly lower in the male partners of RPL couples.

\section{Pairings of HLA-C1/C2 supra-groups in male partners and KIR genes in female partners}

The correlations between the HLA-C1 and HLA-C2 supra-groups in male partners and the KIR genes in female partners were investigated in RPL and healthy couples. The presence of a C1 group ligand
(2DL3) in the female partners that corresponded to the HLA-C2 group in their partners was found to be more common in the RPL couples than in the healthy couples ( $44 \%$ vs. $20 \%, p=0.02$ ).

Since one of the fetal HLA-C alleles is from the mother, the KIR genotype and $\mathrm{HLA}-\mathrm{C} 1 / \mathrm{C} 2$ groups of mothers were matched and evaluated based on the possibility that the mother's HLA-C1/C2 group could also be important for immune behavior during pregnancy. The frequencies of $\mathrm{HLA}-\mathrm{C} 2 / \mathrm{C} 2$ and $\mathrm{HLA}-\mathrm{C} 2 / \mathrm{x}$ genotypes in mothers and their KIR genotypes were compared. KIR2DL2, an HLA-C2 ligand, cooccurred with the $\mathrm{C} 2 / \mathrm{C} 2$ or $\mathrm{C} 2 / \mathrm{x}$ in $62.5 \%$ in the female partners of RPL couples, but only in $20 \%$ of their healthy counterparts ( $p=0.02$ ). No relationship was found between the KIR genotype of female partners and HLA-Bw4/Bw6 homozygosity or heterozygosity in male partners.

\section{Discussion}

Genetic diversity in the evolutionary process is important for the continuation of species. The genes with the highest level of genetic polymorphism in humans are HLA genes. Spousal selection may be associated with this diversity in HLA antigens [37]. Each individual's HLA antigens are unique, and immune responsiveness or tolerance created during the lifetime is mediated by HLAs. HLA compatibility between spouses has been claimed to be advantageous for successful birth after oocyte donation [7]. From this point of view, we examined the compatibility of HLA groups between partners of RPL couples with the hypothesis that between-partner HLA compatibility or incompatibility in RPL couples may be associated with RPL. However, there was no significant difference in the frequency of fully matched (10/10) or incompatible HLAs between RPL and healthy control cou- 
ples. When the couples were grouped according to the number of compatible alleles, a statistically significant difference was found between the groups in terms of the frequency of couples with 5-10 compatible HLA-A/B/C/DR/DQ alleles (Figure 1A). Mismatches in 5-10 alleles were more prominent within RPL couples. This difference may indicate the necessity of a certain level of between-partner HLA compatibility, which may contribute to immune tolerance that allows the development of a healthy semi-allogenic fetus.

HLA compatibility alone between partners is not sufficient to determine the fate of the fetus. Cellular interactions at the maternal-fetal interface are important for healthy fetal development. The maternal NK cell response to the semi-allogeneic fetus occurs in the presence of paternal antigens. Although the relationship between the $\mathrm{HLA} / \mathrm{KIR}$ allele repertoire of the father and mother and the likelihood of abortion has not been clearly defined, it has been emphasized that some HLA alleles may be associated with recurrent abortions [16].

In the light of new findings, other immunological factors associated with HLA alleles have become more prominent than the frequency of classical HLA alleles. Although the data are preliminary and varying, it has been suggested that MHC-KIR genes or molecules may play a role in recurrent miscarriages [32]. KIR-HLA-Bw4/Bw6 and KIR-HLA-C interactions have been shown to mediate chronic rejection reactions after kidney transplantation, although the ligand interactions of KIRs involved in NK cell function remain unclear [29,38]. We suggest that similar interactions might be implicated in RPL. It is known that extravillous cytotrophoblast cells express only classical HLA-C and non-classical HLA-E and HLA-G in locations where the semi-allogeneic fetus is trying to develop [9]. Although HLA-B expression in extravillous trophoblasts is unknown, Shankarkumar et al. [16] found that $B * 57: 01$ was significantly higher in RPL patients. In cases of RPL, although there is no known expression of HLA-B in extravillous trophoblasts, other HLA class I molecules have also been shown to be inducible by agents such as human cytomegalovirus [39]. The behavior of extravillous trophoblasts in cases of RPL with unknown pathogenesis may differ from normal pregnancy [40]. In our study, we found a significant level of HLA-B mismatching between partners in RPL couples, but the mismatching may not have been related to Bw4/Bw6 homozygosity or heterozygosity as KIR ligands, even though more prominent mismatches were observed in the presence of Bw4 antigens. A potential reason for this may have been polymorphisms in KIR3DL1 (a Bw4 receptor), which we could not evaluate since KIR3DL1 was present in $100 \%$ of the subjects analyzed in this study.

We also examined KIR gene frequencies. In previous studies of KIR gene repertoires, it was found that women with recurrent miscarriages had fewer inhibitory KIR genes than control women who ex- perienced healthy childbirth [41]. However, our results did not confirm that finding. In contrast, decreased expression of an inhibitory KIR receptor (KIR2DL2) has been reported in women with RPL, and the importance of the interactions between KIR molecules and the HLA-C1/C2 subgroups has been pointed out [42,43]. In our study, we did not find a significantly lower KIR2DL2 allele frequency in the female partners of RPL couples. Nonetheless, interestingly, the KIR2DL5 allele frequency in our study group was significantly higher in both the female $(p=0.03)$ and male $(p=0.001)$ partners of RPL couples than in healthy controls. The KIR2DS3 gene allele frequency was significantly lower in men with RPL $(p=0.001)$. In contrast to our findings, Hiby et al. [44] found that the frequency of KIR2DL5 was lower in mothers with preeclampsia than in healthy controls, and also reported that the interaction between the maternal AA haplotype and the fetal HLA-C2 group may be a risk factor for the fetus. Although there was no significant difference in KIR haplotypes between RPL and healthy couples in our study, we found a statistically significant trend towards $\mathrm{C} 2$ homozygosity in the male partners of RPL couples, which might be a risk factor for the allogeneic fetus. However, the frequency of KR2DL1, a known ligand of HLA-C2, was excluded from our statistical analysis since it was detected in $100 \%$ of the sample of the general Turkish population and the RPL couples in our study [35]. Further studies to establish the genetic variants of KIR2DL1 linked to RPL would provide valuable information on the interactions between HLA-C2 and HLA-C2 ligands.

In our study, a high frequency of KIR2DL5 was found in men and women in RPL couples, while KIR2DS3 was less common in the male partners of couples with RPL. These genes are related and their ligands are not known [26]. The presence of the KIR2DL3 gene in women with RPL corresponded to the presence of the HLA-C2 group in their male partners to a statistically significant extent in comparison with the controls $(p=0.03)$. Statistical significance for this relationship was not found in healthy couples $(p=0.6)$. Controlled activation of immune system and balanced cell growth may be of importance for a healthy pregnancy. The healthy development of the fetus during pregnancy requires controlled activation of immune system cells, especially at the maternal-fetal interface [45]. According to our findings, the co-occurrence of the KIR2DL3 inhibitory receptor gene in women with RPL with the presence of the HLA-C2 ligand in their spouses may be interpreted as reflecting the absence of optimal activation of NK cells in these women.

The ligand of KIR2DL2 is HLA-C1. In the RPL group, women with KIR2DL2 and HLA-C2/C2 or HLA-C2/x genotypes were significantly more common than in the healthy group. Therefore, the KIR-HLA C distribution in women with RPL is as important as concordance between partners, and this finding is compatible with the data reported by the Higgs group. It is obvious that KIRs in men cannot play a direct 
role in the abortion process, but since the KIR distribution of the fetus is unknown, scattered fetal cells bearing possible unacceptable KIRs may have an effect on accelerating the termination process. Furthermore, the observation of different frequencies of KIR2DL5 and KIR2DS3, which are orphan genes and receptors, also suggests that their difference may vary across ethnicities. Identifying the ligands of the KIR2DL5 and KIR2DS3 receptors, which are highly homologous with each other, determining their status in healthy and pathological conditions, and demonstrating their relationship with RPL will contribute greatly to the literature on this topic.

Finally, we suggest that multiple distinct mechanisms may underlie the HLA-KIR interactions in RPL that may lead to the disruption of individual immune recognition. The elucidation of the factors affecting these mechanisms may help to explain contradictory data in the literature from couples drawn from various ethnic populations.

\section{Conflict of interest}

No potential conflict of interest relevant to this article was reported.

\section{ORCID}

$\begin{array}{ll}\text { Mehmet Onur Elbaşı } & \text { https://orcid.org/0000-0002-9851-9796 } \\ \text { Aysın Tulunay } & \text { https://orcid.org/0000-0003-4735-9902 } \\ \text { Hale Karagözoğlu } & \text { https://orcid.org/0000-0001-7895-8778 } \\ \text { Semra Kahraman } & \text { https://orcid.org/0000-0002-2686-4240 } \\ \text { Emel Ekşioğlu-Demiralp } & \text { https://orcid.org/0000-0002-7695-0614 }\end{array}$

\section{Author contributions}

Conceptualization: HK, SK, EED. Data curation: MOE, AT. Formal analysis: MOE, AT, EED. Methodology: HK, SK, EED. Project administration: MOE, EED. Visualization: EED. Writing-original draft: MOE, EED. Writing-review \& editing: SK, EED.

\section{References}

1. ESHRE Guideline Group on RPL, Bender Atik R, Christiansen OB, Elson J, Kolte AM, Lewis S, et al. ESHRE guideline: recurrent pregnancy loss. Hum Reprod Open 2018;2018:hoy004.

2. Kline JS. Very early pregnancy. Reprod Toxicol 1985;(1985):25165.

3. Coulam CB. Understanding the immunobiology of pregnancy and applying it to treatment of recurrent pregnancy loss. Early Pregnancy 2000;4:19-29.

4. Christiansen OB. A fresh look at the causes and treatments of recurrent miscarriage, especially its immunological aspects. Hum
Reprod Update 1996;2:271-93.

5. Ford HB, Schust DJ. Recurrent pregnancy loss: etiology, diagnosis, and therapy. Rev Obstet Gynecol 2009;2:76-83.

6. Ober C. Studies of HLA, fertility and mate choice in a human isolate. Hum Reprod Update 1999;5:103-7.

7. Lashley LE, Haasnoot GW, Spruyt-Gerritse M, Claas FH. Selective advantage of HLA matching in successful uncomplicated oocyte donation pregnancies. J Reprod Immunol 2015;112:29-33.

8. Faulk WP, Temple A. Distribution of beta2 microglobulin and HLA in chorionic villi of human placentae. Nature 1976;262:799802.

9. Sargent IL, Borzychowski AM, Redman CW. NK cells and human pregnancy: an inflammatory view. Trends Immunol 2006;27:399404.

10. Moffett A, Loke C. Immunology of placentation in eutherian mammals. Nat Rev Immunol 2006;6:584-94.

11. Moffett-King A. Natural killer cells and pregnancy. Nat Rev Immunol 2002;2:656-63.

12. McIntyre JA, Faulk WP. Recurrent spontaneous abortion in human pregnancy: results of immunogenetical, cellular, and humoral studies. Am J Reprod Immunol 1983;4:165-70.

13. Wagenknecht DR, Green KM, Mclntyre JA. Analyses of HLA-DQ alleles in recurrent spontaneous abortion (RSA) couples. Am J Reprod Immunol 1997;37:1-6.

14. Sbracia M, Mastrone M, Scarpellini F, Grasso JA. Influence of histocompatibility antigens in recurrent spontaneous abortion couples and on their reproductive performances. Am J Reprod Immunol 1996;35:85-92.

15. Kruse C, Steffensen R, Varming K, Christiansen OB. A study of HLADR and -DQ alleles in 588 patients and 562 controls confirms that HLA-DRB1*03 is associated with recurrent miscarriage. Hum Reprod 2004;19:1215-21.

16. Shankarkumar U, Pawar A, Gaonkar P, Parasannavar D, Salvi V Ghosh K. HLA allele associations in idiopathic recurrent spontaneous abortion patients from India. J Hum Reprod Sci 2008;1:1924.

17. Aagaard-Tillery KM, Silver R, Dalton J. Immunology of normal pregnancy. Semin Fetal Neonatal Med 2006;11:279-95.

18. Clarke AG, Kendall MD. The thymus in pregnancy: the interplay of neural, endocrine and immune influences. Immunol Today 1994;15:545-51.

19. Lanier LL. NK cell recognition. Annu Rev Immunol 2005;23:22574.

20. Kumar V, McNerney ME. A new self: MHC-class-l-independent natural-killer-cell self-tolerance. Nat Rev Immunol 2005;5:36374.

21. Vivier E, Anfossi N. Inhibitory NK-cell receptors on T cells: witness 
of the past, actors of the future. Nat Rev Immunol 2004;4:190-8.

22. Middleton D, Curran M, Maxwell L. Natural killer cells and their receptors. Transpl Immunol 2002;10:147-64.

23. Parham P. MHC class I molecules and KIRs in human history, health and survival. Nat Rev Immunol 2005;5:201-14.

24. Moretta L, Moretta A. Killer immunoglobulin-like receptors. Curr Opin Immunol 2004;16:626-33.

25. Moesta AK, Parham P. Diverse functionality among human NK cell receptors for the C1 epitope of HLA-C: KIR2DS2, KIR2DL2, and KIR2DL3. Front Immunol 2012;3:336.

26. Ordonez D, Meenagh A, Gomez-Lozano N, Castano J, Middleton $D$, Vilches C. Duplication, mutation and recombination of the human orphan gene KIR2DS3 contribute to the diversity of KIR haplotypes. Genes Immun 2008;9:431-7.

27. Cisneros E, Moraru M, Gomez-Lozano N, Lopez-Botet M, Vilches C. KIR2DL5: an orphan inhibitory receptor displaying complex patterns of polymorphism and expression. Front Immunol 2012; 3:289.

28. Muller CA, Engler-Blum G, Gekeler V, Steiert I, Weiss E, Schmidt H. Genetic and serological heterogeneity of the supertypic HLA-B locus specificities Bw4 and Bw6. Immunogenetics 1989;30:2007.

29. Prakash S, Sarangi AN, Alam S, Sonawane A, Sharma RK, Agrawal S. Putative role of KIR3DL1/3DS1 alleles and HLA-Bw4 ligands with end stage renal disease and long term renal allograft survival. Gene 2017;637:219-29.

30. Grifoni A, Montesano C, Patronov A, Colizzi V, Amicosante M. Immunoinformatic docking approach for the analysis of KIR3DL1/ HLA-B interaction. Biomed Res Int 2013;2013:283805.

31. Lutz CT. Human leukocyte antigen Bw4 and Bw6 epitopes recognized by antibodies and natural killer cells. Curr Opin Organ Transplant 2014;19:436-41.

32. Moffett A, Chazara O, Colucci F, Johnson MH. Variation of maternal KIR and fetal HLA-C genes in reproductive failure: too early for clinical intervention. Reprod Biomed Online 2016;33:763-9.

33. Eksioglu Demiralp E, inventors. A method for fast and high-quality genomic or cell fee DNA isolation in a single tube and, a related kit. Turkey patent 2019/015222019-GE-44483. 2019. TURKEY patent 2019/015222019-GE-44483. 2019.

34. Gonzalez-Galarza FF, Takeshita LY, Santos EJ, Kempson F, Maia $\mathrm{MH}$, da Silva AL, et al. Allele frequency net 2015 update: new features for HLA epitopes, KIR and disease and HLA adverse drug reaction associations. Nucleic Acids Res 2015;43:D784-8.

35. Elbasi MO, Tulunay Virlan A, Eksioglu Demiralp E. Killer cell immunoglobulin-like receptor genotypes in the Turkish population. Hum Immunol 2018;79:193-4.

36. Peakall R, Smouse PE. Genalex 6: genetic analysis in Excel. Population genetic software for teaching and research Molecular Ecology Notes 2006;6:288-95.

37. Edwards SV, Hedrick PW. Evolution and ecology of MHC molecules: from genomics to sexual selection. Trends Ecol Evol 1998; 13:305-11.

38. Littera R, Piredda G, Argiolas D, Lai S, Congeddu E, Ragatzu P, et al. KIR and their HLA Class I ligands: two more pieces towards completing the puzzle of chronic rejection and graft loss in kidney transplantation. PLoS One 2017;12:e0180831.

39. Terauchi M, Koi H, Hayano C, Toyama-Sorimachi N, Karasuyama $\mathrm{H}$, Yamanashi $\mathrm{Y}$, et al. Placental extravillous cytotrophoblasts persistently express class I major histocompatibility complex molecules after human cytomegalovirus infection. J Virol 2003; 77:8187-95.

40. Windsperger K, Dekan S, Pils S, Golletz C, Kunihs V, Fiala C, et al. Extravillous trophoblast invasion of venous as well as lymphatic vessels is altered in idiopathic, recurrent, spontaneous abortions. Hum Reprod 2017;32:1208-17.

41. Varla-Leftherioti M, Spyropoulou-Vlachou M, Niokou D, Keramitsoglou T, Darlamitsou A, Tsekoura C, et al. Natural killer (NK) cell receptors' repertoire in couples with recurrent spontaneous abortions. Am J Reprod Immunol 2003;49:183-91.

42. Flores AC, Marcos CY, Paladino N, Arruvito L, Williams F, Middleton $D$, et al. KIR receptors and HLA-C in the maintenance of pregnancy. Tissue Antigens 2007;69 Suppl 1:112-3.

43. Sharkey AM, Gardner L, Hiby S, Farrell L, Apps R, Masters L, et al. Killer lg-like receptor expression in uterine NK cells is biased toward recognition of HLA-C and alters with gestational age. J Immunol 2008;181:39-46.

44. Hiby SE, Walker JJ, O'shaughnessy KM, Redman CW, Carrington $M$, Trowsdale J, et al. Combinations of maternal KIR and fetal HLA-C genes influence the risk of preeclampsia and reproductive success. J Exp Med 2004;200:957-65.

45. Mor G, Cardenas I. The immune system in pregnancy: a unique complexity. Am J Reprod Immunol 2010;63:425-33. 